\title{
Review: Genetic diversity studies using microsatellite markers and their contribution in supporting sustainable sheep breeding programs
}

\author{
OUMER SHERIFF ${ }^{1, \boldsymbol{v}}$, KEFYALEW ALEMAYEHU ${ }^{2}$ \\ ${ }^{1}$ Department of Animal Science, College of Agriculture and Natural Resources, Assosa University. P.O.Box 18, Assosa, Ethiopia. "email: \\ soumer74@yahoo.com \\ ${ }^{2}$ Department of Animal Production and Technology, College of Agriculture and Environmental Sciences, Bahir Dar University. P.O.Box 79, Bahir Dar, \\ Ethiopia
}

Manuscript received: 1 May 2017. Revision accepted: 28 June 2017.

\begin{abstract}
Sheriff O, Alemayehu K. 2017. Review: Genetic diversity studies using microsatellite markers and their contribution in supporting sustainable sheep breeding programs. Asian J Agric 1: 46-51. Microsatellites have been widely accepted and employed as useful molecular markers for measuring genetic diversity and divergence within and among populations. The various parameters developed so far to measure genetic diversity within and among populations are observed and expected heterozygosity (Ho and He), the mean number of alleles per locus (MNA), polymorphic information content (PIC), genetic distance and phylogenetic or tree building approach. The objective of this review was therefore to quantify the genetic diversity studies of domestic sheep populations using microsatellite markers and their contribution in supporting sustainable sheep breeding programs. From the review, it is possible to see that there were high population genetic variations in all the studied sheep populations, poor levels of population differentiation and high levels of inbreeding. On the other hand, low estimates of heterozygosity and mean number of alleles and employing only few and weak markers were observed in some of the studies. The gaps observed in the previous genetic diversity studies of the sheep populations may demand further works to reveal more information on the population structures and to start appropriate and sustainable breeding programs.
\end{abstract}

Keywords: Genetic diversity, microsatellites, sheep, sustainable breeding

Abbreviations: DA: Cavalli-Sforza genetic distance, DS: Nei's standard genetic distance, FAO: Food and agricultural organization of the united nations, FIS: Level of inbreeding, FST: Genetic differentiation between subpopulations, He: Expected heterozygosity, Ho: Observed heterozygosity, HWE: Hardy-Weinberg equilibrium, MNA: Mean number of alleles per locus, PIC: Polymorphic information content

\section{INTRODUCTION}

Domestic farm animals are crucial for food and agriculture, providing 30 to 40 percent of the agricultural sector's global economic value (FAO 2000). Despite their invaluable contribution to the global economy, there is a rapid loss of genetic resources in farm animals and the world loses two breeds of its valuable domestic diversity every week (FAO 2000). Hence, there should be an urgent mechanism to maintain and document the diversity of livestock genetic resources and design appropriate strategies for conservation and sustainable use, particularly in developing countries (Hanotte and Jialin 2005).

Maintenance of livestock genetic diversity is a key to the long-term survival of most species and should be done based on comprehensive information regarding the structure of the populations, including sources of genetic variability within and among populations. It also requires adequate implementation of conservation priorities and sustainable management programs (Mahmoudi et al. 2011) to be widely used to categorize livestock species in the world (Cardellino and Boyazoglu 2009).

Genetic diversity (the variation of alleles and genotypes present in a population) provides a basis for adaptive and evolutionary processes (Frankham et al. 2002). The current pool of diversity in livestock has been created by the forces of both natural and artificial selection (Groeneveld et al. 2010). These forces encompass processes such as mutations, adaptations, segregation, selective breeding, and genetic drift (Groeneveld et al. 2010). Future generations of domesticated species are wholly dependent on genetic variation which will be observed from genetic differences between breeds, between populations within a breed and between individuals within a population (Groeneveld et al. 2010).

Globally, sheep have the highest number of recorded breeds, contributing $25 \%$ to the total mammalian breeds adapted to a broad range of environments (Gizaw et al. 2008). The adaptation of different breeds to a broad range of agroecology provides the necessary variability that offers opportunities to meet the increased future demands for food and provide flexibility to respond to changing markets and needs (Wollny 2003). To date, more than 1078.2 million sheep populations are kept in different parts of the world with the following share in million: Asia (452.3), Africa (287.6), Northern America (6.9), Central 
America (8.1), Caribbean (3.1), South America (73.1), Europe (133.9) and Oceania (113.1) (Mahmoud, 2010).

Microsatellites have been widely accepted as useful tools for measuring genetic diversity and divergence within and among populations (FAO 2011). So far, several genetic diversity studies on sheep have been conducted using microsatellite markers (Adamov et al. 2011). Their abundance, high level of repeat-number polymorphism, manifested as the occurrence of many alleles per locus, and co-dominant inheritance has facilitated their extensive use in genome mapping, phylogenetic inference, and population genetics in farm animals (FAO 2011). However, most of the genetic diversity studies of sheep using microsatellite markers, conducted so far, may not be as supportive as expected in revealing the required information for designing appropriate and sustainable sheep breeding programs and conservation strategies. Therefore, the objective of this review was to quantify the genetic diversity studies using microsatellite markers and their contribution in supporting sustainable sheep breeding programs.

\section{GENETIC DIVERSITY AND WITHIN POPULATION VARIATION}

Some of the parameters which can help to study genetic diversity within a population are the mean number of alleles per locus (MNA), the average expected and observed heterozygosity values (Halima et al. 2012b). Additionally, testing for deviations from the HardyWeinberg equilibrium (HWE) per population gives insight about those primary forces viz., natural selection, mutation, genetic drift, nonrandom mating, and genetic migration that derive evolutionary change (Ojango et al. 2011). On the other hand, the precision of estimated genetic diversity is a function of the number of loci analyzed, the heterozygosity of these loci and the number of animals sampled in each population (Barker 1994).

\section{ESTIMATION OF MEAN NUMBER OF ALLELES (MNA)}

The mean number of alleles is a good indicator of the genetic polymorphism within the population (Halima et al. $2012 \mathrm{~b}$ ) and it depends on sample size of the population because of the potential presence of unique alleles in a population that may occur at low frequencies (Sithembile 2011). The number of detected alleles may increase with an increase in population size. A high number of alleles imply more genetic variation (Nei 1987). Mean number of alleles that indicate the genetic polymorphism within the studied microsatellites were reported for several sheep populations (Table 1).

The mean number of alleles (MNAs) (Table 1) showed relatively lower estimates for some Ethiopian, Chinese, and South African sheep populations. For the other sheep populations, relatively encouraging estimates of MNA were reported. A high number of alleles imply more genetic variation (Nei 1987), and it is the key relevance in conservation programs. However, though those reports explain the existence of high polymorphism, the average number of alleles depends on sample size; number of observed alleles tends to increase with increasing population size (Aljumaah et al. 2012). Therefore, it is important to compare sample population sizes that are close to equal (Sithembile 2011). However, some of the studies used not only very small number of animals which is quite far from FAO recommendation for microsatellite marker analysis (FAO 2011), e.g., Hirbo et al. 2006 used only 9 animals to represent a population, but also, they used unequal sample size. This may lead to biasedness in estimating genetic parameters such as HWE and MNA, additionally, there was not any technique indicated in the papers which were employed to handle such a limitation.

It was observed that most of the sheep genetic diversity studies (Table 1) were undertaken by using few numbers of microsatellite markers. All 30 microsatellites, the maximum coverage recommended by FAO (2011), were covered only for Merino derived and Albanian sheep breeds (Ceccobelli et al. 2009; Hoda and Marsan 2012). Genetic diversity studies with a greater number of microsatellite markers, not only reveal more information on the population structures but also offer more opportunities to compare with results from previous studies undertaken with various subsets of the markers (FAO 2011).

\section{ESTIMATION OF OBSERVED (HO) AND EXPECTED (HE) HETEROZYGOSITIES}

Observed heterozygosity, the proportion of heterozygotes observed in a population, and expected heterozygosity, the percentage of loci heterozygous per individual or the number of individuals heterozygous per locus (Ojango et al. 2011) are the most widely used parameters to measure genetic diversity in a population (Toro et al. 2009). Literature suggests that heterozygosity estimates having greater than 0.5 heterozygosity values are believed to be appropriate for genetic diversity studies (Davila et al. 2009; Dorji et al. 2012). However, the heterozygosity estimates observed in some Indian, South African, Ethiopian, Chinese, Chilean, Kenyan and Nigerian sheep populations (Table 1) were below 0.5 or closer to the margin. These low heterozygosity estimates might be due to maintaining microsatellite loci that had registered values below 0.5 in the respective breeds during the analysis. On the other hand, very low heterozygosity estimates maybe because of the effect of small population size, high selection pressure in closed population, inbreeding and minimal or null immigration of new genetic materials into the population (Canon et al. 2006). The heterozygosity (both observed and expected) estimates in the remaining sheep populations are relatively high, concluding that the studied sheep populations have high amount of withinpopulation genetic diversity. 
Table 1. Estimated heterozygosity, mean number of alleles, polymorphic information content and level of inbreeding

\begin{tabular}{|c|c|c|c|c|c|c|c|c|}
\hline Breed & $\begin{array}{l}\text { Country of } \\
\text { origin }\end{array}$ & $\mathrm{He}$ & Ho & MNA & $\begin{array}{l}\text { PIC per } \\
\text { locus }\end{array}$ & FIS & $\begin{array}{l}\text { MS } \\
\text { (No.) }\end{array}$ & Author \\
\hline Vembur sheep & India & 0.73 & 0.52 & 5.88 & 0.69 & 0.29 & 25 & Pramod et al. (2011) \\
\hline Kail sheep & India & 0.72 & 0.77 & 5.27 & 0.60 & 0.053 & 11 & Ahmed et al. (2014) \\
\hline sheep breeds (7) & South Africa & 0.63 & 0.45 & $5-16$ & 0.95 & NA & 24 & Buduram (2004) \\
\hline Turkish breeds (4) & Turkey & 0.87 & 0.66 & 7.04 & NA & 0.07 & 17 & Yilmaz et al. (2015) \\
\hline Turkish native and cross sheep (11) & Turkey & 0.75 & 0.72 & $5.8-11.8$ & NA & $0.09-0.16$ & 15 & Evren (2004) \\
\hline traditional sheep populations (14) & Ethiopia & $0.66-0.75$ & NA & 6.79 & NA & FST $(0.046)$ & 17 & Gizaw (2008) \\
\hline sheep breeds (3) & Ethiopia & 0.50 & 0.33 & $3-23$ & 0.69 & 0.236 & 22 & Nigussie (2015) \\
\hline Italian merino derived sheep (3) & Italy & $0.64-0.75$ & $0.61-0.70$ & $5.17-8.43$ & NA & $0.048-0.118$ & 30 & Ceccobelli et al. (2009) \\
\hline Pelt sheep (3) & Iran & 0.83 & 0.99 & 7.6 & 0.81 & -0.19 & 15 & Hatami et al. (2014) \\
\hline Local Sheep (8) & China & 0.54 & 0.59 & $3.8-5.4$ & 0.49 & 0.404 & 10 & Zeng et al. (2010) \\
\hline Albanian Sheep (3) & Albania & 0.75 & 0.72 & 8.54 & 0.72 & 0.041 & 31 & Hoda and Marsan (2012) \\
\hline Chilean sheep (4) & Chile & 0.82 & 0.696 & $9-25$ & $0.55-0.90$ & 0.040 & 9 & De la Barra et al. (2010) \\
\hline Sheep populations (15) & Kenya & 0.72 & 0.65 & 7.70 & NA & 0.109 & 15 & Mukhongo et al. (2014) \\
\hline Nigerian Indigenous Sheep (4) & Nigeria & 0.78 & 0.49 & 8.64 & 0.85 & 0.34 & 15 & Brilliant et al. (2012) \\
\hline Sheep breeds (3) & Saudi Arabia & $0.59-0.82$ & $0.65-0.989$ & 11.47 & 0.75 & 0.031 & 17 & Mahmoud et al. (2017) \\
\hline $\begin{array}{l}\text { Trans-caucasian, Asian, European and African } \\
\text { sheep breeds (22) }\end{array}$ & $*$ & $0.62-0.81$ & $0.60-0.77$ & $6.71-9.36$ & NA & FST $(0.06-0.10)$ & 14 & Hirbo et al. (2006) \\
\hline Karakul sheep & Iran & 0.831 & 0.989 & 8.07 & 0.81 & -0.197 & 15 & Nanekarani et al. (2011) \\
\hline Romanian sheep breeds (4) & Romania & 0.740 & 0.640 & 9.275 & NA & NA & 11 & Emil and Marieta 2012) \\
\hline NamaquaAfrikaner (3) & South Africa & 0.50 & 0.49 & 3.9 & 0.44 & 0.019 & 22 & Sithembile (2011) \\
\hline Sheep breeds (10) & $* *$ & 0.74 & 0.59 & $5.4-6.0$ & NA & 0.060 & 10 & Farid et al. (2000) \\
\hline
\end{tabular}

Note: MS = Microsatellite; * Azerbajan (5), Armeni

Canada, Iceland, USA, Denmark, UK and Kenya 
Most of the observed heterozygosity values are generally closer to, but lower than, the expected heterozygosity in most of the breeds and loci indicating no overall loss in heterozygosity (allele fixation) (Araujo et al. 2006) and the populations are at Hardy-Weinberg equilibrium (HWE).

\section{ESTIMATION OF POLYMORPHIC INFORMATION CONTENT (PIC)}

Polymorphic information content (PIC) depicts the suitability of the markers and their primers used in the study for analyzing the genetic variability of a population. A marker with PIC $>0.5$ can be considered as highly informative and highly polymorphic, whereas $0.5>$ PIC $>0.25$ is recognized as reasonably informative and below 0.25 is measured as slightly informative (Marshall et al. 1998). In line with this, highly polymorphic markers were employed for most of the sheep populations studied (Table 1) except the local sheep breeds in China $\mathrm{PIC}=0.492$. In fact, PIC is determined by heterozygosity and number of alleles (Aljumaah et al. 2012) and this makes microsatellite markers the choice for genetic characterization and diversity studies.

\section{LEVEL OF INBREEDING (FIS)}

FIS is estimated for populations that show significant deviation from the HWE and are significant for significant HWE estimation (Ojango et al. 2011). A high positive FIS indicates a high degree of homozygosity and vice versa, while negative values indicate low level of inbreeding (Dorji et al. 2012). Taking this background information into consideration, moderate to high inbreeding levels were reported by various scholars for different sheep populations; for instance, three sheep breeds of Ethiopia $(F I S=0.236)($ Nigussie 2015), Vembur $(F I S=0.29)($ Pramod et al. 2011), Magra ( $F I S=0.159)$ (Arora and Bhatia 2006) and Kheri $(F \mathrm{IS}=0.128$ ) (Arora and Bhatia 2006)sheep breeds of India, some Merino derived sheep breeds of Italy ( $F \mathrm{IS}=0.048-0.118)$ (Ceccobelli et al. 2009), some Turkish sheep breeds ( $F \mathrm{IS}=0.09-0.16)$ (Evren 2004) eight local sheep breeds of China $(F I S=0.404)$ (Zeng et al. 2010), fifteen sheep populations of Kenya $(F \mathrm{IS}=0.109)$ (Mukhongo et al. 2014) and Nigerian indigenous sheep (FIS=0.34) (Brilliant et al. 2012). This might be because of the small sheep population size, closed breeding system and very limited number of breeding rams used for many consecutive years. The lowest heterozygosity and MNA estimates indicated in table 1 above strengthen this justification.

However, tolerable mean values of FIS (0.087) for Ganjam (Arora et al. 2010), (0.0525) for Kail (Ahmed et al. 2014) and (0.0786) for Tamil Nadu (Kavitha et al. 2010) sheep breeds of India and FIS (0.07) for Turkish breeds (Yilmaz et al. 2015) were reported by scholars. These moderate levels of inbreeding may be a result of moderate levels of mating between closely related individuals under field conditions and maybe the uncontrolled and unplanned mating that caused high levels of inbreeding (Mekuriaw et al. 2016). On the contrary, FIS (-0.19) (Hatami et al. 2014) and FIS (-0.197) (Nanekarani et al. 2011) depict low levels of inbreeding and an excess of heterozygotes was reported for three Iranian sheep breeds and Karakul sheep breed of Iran, respectively.

\section{GENETIC DISTANCE AND VARIATION AMONG POPULATIONS}

Kalinowski (2004) had suggested that the highest genetic distance (FST) be higher than 0.25 , moderate to be between 0.05 and 0.25 and the lowest estimate below 0.05 . In relative to many reports, the genetic distance among most of the populations obtained by many of the scholars (Farid et al. 2000 (FST=0.163); Evren 2004 (FST=0.0020.146); Hirbo et al. 2006 ( $F S T=-0.001-0.183$ ); Sithembile 2011 (FST=0.105); Brilliant et al. 2012 ( $F \mathrm{ST}=0.088)$; Hoda and Marsan 2012 ( $F S T=0.011$ ); Hatami et al. 2014 $(F \mathrm{ST}=0.018)$; Mukhongo et al. $2014 \quad(F S T=0.101)$ and Mahmoud et al. $2017(F S T=0.042))$ is almost negligible $(<0.05)$ and/or moderate $(0.05<F S T<0.25)$ values. Some of the authors revealed significant genetic distance estimates among populations. This implies that there is relatively low to moderate genetic sub-differentiation among the sheep populations. A fixation index (FST) of about 0.15 is an indication of significant differentiation among populations (Frankham et al. 2002).

The average FST value overall microsatellite loci in the sheep populations in Ethiopia was reported to be 0.046 , indicating a $4.6 \%$ of total genetic variation among populations and a $95.4 \%$ difference among individuals (Gizaw 2008). The same author reported that lack of differentiation in those phenotypically different subpopulations could be due to gene flow between the areas having close geographical distance and similar ecology. Similarly, Nigussie (2015) noted that 3\% of the total variation occurred due to population subdivision, while 97\% of the variation existed among individuals within the sheep populations, which might be due to migration of individuals from one sub-population to the other (Nigussie 2015). Hailu et al. (2008) and Halima et al. (2012b) also confirmed that the low genetic differentiation between subpopulations might be due to traditional uncontrolled mating practices and policies that facilitated or led to uncontrolled movement of animals through various market routes and agricultural extension systems in Ethiopia.

\section{IDENTIFIED GAPS, THEIR IMPLICATIONS AND FUTURE PROSPECTS}

One of the gaps, identified so far, is related to the expected and observed heterozygosity estimates and microsatellite loci. It is generally suggested that microsatellite loci showing $\mathrm{He}$ and $\mathrm{Ho}$ estimates of less than 0.5 were not appropriate for heterozygosity evaluation. However, microsatellite loci with 
heterozygosity estimate less than 0.5 or close to that were used in some of the studies (Table 1).

Similarly, though FAO (2011) recommended the genetic diversity studies of livestock using all the 30 microsatellite markers, most of the sheep genetic diversity studies were undertaken by using a subset of the markers. For example, De la Barra et al. (2010) used only 9 microsatellites to study four Chilean sheep breeds and Farid et al. (2000) used only 10 microsatellites to study ten sheep populations in Canada, Iceland, USA, Denmark, UK, and Kenya. Hence, studying a greater number of microsatellite markers to reveal more information on the population structure is suggested in future sheep genetic diversity studies. If less than 30 microsatellites are to be used, it is important to be keen in selecting microsatellites to bring an appropriate recommendation that can support sustainable breeding strategies.

The mean number of alleles (MNA) in sheep genetic diversity studies in Ethiopia, China, and South Africa (Zeng et al. 2010; Sithembile 2011; Nigussie 2015) were below the recommended value, the microsatellite loci for genetic diversity studies should have more than four alleles (FAO 2011). This indicated that some of the microsatellite loci were not sufficiently polymorphic and were not appropriate for genetic diversity analysis.

Some of the diversity studies used not only very small number of sheep which is by far lower than the recommendation of FAO for microsatellite marker analysis (FAO 2011), e.g., Hirbo et al. 2006 used only 9 animals to represent a population, but also, they used unequal sample size. This may lead to biasedness in estimating genetic parameters such as the MNA, there was not any technique indicated in the papers which were employed to handle such a limitation.

All these gaps point out that the microsatellites which were not sufficiently polymorphic could be dropped out and it is very important to be ardent in selecting them to bring the right recommendation that can support appropriate and sustainable sheep breeding programs.

\section{CONCLUDING REMARK}

The results from this review indicated that the within population genetic diversity, in all sheep populations, is extremely higher than between population variation which might be due to the uncontrolled and random mating practiced among the breeding flocks having close geographical distance and similar ecology. There was also poor level of population differentiations, high levels of inbreeding, low estimates of heterozygosity and MNA and markers which were not sufficiently polymorphic in most of the studies. All these results demand further works to reveal more information on the sheep population structures and help to start sustainable breeding programs and policies involving the decision on pure or crossbreeding. Moreover, appropriate conservation activities on breeding farms must be taken to avoid losses of genetic diversity and thereby to support the breeding programs. It is also suggested to set up an improvement scheme for the frequent exchange of rams among farms or flocks rearing the same breed, aimed to increase genetic diversity.

\section{ACKNOWLEDGEMENTS}

The first author gratefully acknowledges the Federal Ministry of Education, Ethiopia, Assosa and Bahirdar Universities for the Ph.D. fellowship award.

\section{REFERENCES}

Adamov N, Mickov L, Petkov V, Adamov M. 2011. Microsatellite markers for pedigree verification in cattle. Mac J Anim Sci 1: 9-15.

Ahmed Z, Babar ME, Hussain T, Nadeem A, Awan FI, Wajid A, Shah SA, Ali MM. 2014. Genetic Diversity analysis of Kail Sheep by using microsatellite markers. J Anim Plant Sci 24 (5): 1329-1333.

Aljumaah RS, Musthafa MM, Al-Shaikh MA, Badri OM, Hussein MF. 2012. Genetic diversity of Ardi goat based on microsatellite analysis. African J Biotech 11 (100): 16539-16545.

Araujo AM, Guimarães SEF, Machado TMM, Lopes PS, Pereira CS, da Silva FLR, Rodrigues MT, Columbiano VS. Fonseca CG. 2006. Genetic diversity between herds of Alpine and Saanen dairy goats and the naturalized Brazilian Moxotó breed. J Gen Mol Biol 29 (1): $67-$ 74.

Arora R, Bhatia S, Jain A. 2010. Morphological and genetic characterization of Ganjam sheep. Anim Gen Res 46: 1-9.

Arora R, Bhatia S. 2006. Genetic diversity of Magra sheep from India using microsatellite analysis. Asian-Aust J Anim Sci 19 (7): 938-942.

Barker JSF 1994. A global protocol for determining genetic distances among domestic livestock breeds. In: Gelph V (eds). World Congress on Genetics Applied to Livestock Production 21: 501-508.

Buduram P. 2004. Genetic Characterization of Southern African Sheep breeds using DNA markers. [Thesis]. Department of Animal, Wildlife and Grassland Sciences, Faculty of Agricultural Sciences, University of the Free State, South Africa.

Canon J, Garci'a D, Garcı'a-Atance MA, Obexer-Ruff G, Lenstra JA Ajmone-Marsan P, Dunner S. 2006. The ECONOGENE Consortium. Geographical partitioning of goat diversity in Europe and the Middle East. J Anim Gen 37: 327-334.

Cardellino RA, Boyazoglu J. 2009. Research opportunities in the field of animal genetic resources. Livestock Sci 120: 166-173.

Davila SG, Gil Resino-Talavan MG, Campo JL. 2009. Evaluation of diversity between different Spanish chicken breeds, a tester line and White Leghorn population based on microsatellite markers. Poult Sci 88: 2518-2525.

De la Barra R, Uribe H, Latorre E, San Primitivo F, Arranz J. 2010. Genetic structure and diversity of four Chilean sheep breeds. Chilean J Agric Res 70:646-651.

Dorji N, Duangjinda M, Phasuk Y. (2012). Genetic characterization of Bhutanese native chickens based on an analysis of Red Junglefowl (Gallus gallus gallus and Gallus gallus spadecieus), domestic Southeast Asian and commercial chicken lines (Gallus gallus domesticus). Genet Mol Biol 35 (3): 603-609.

Emil GS, Marieta C. 2012. Genetic characterization of Romanian local breeds using microsatellite markers. In: Caliskan M (ed.). Biochemistry, Genetics and Molecular Biology: Analysis of Genetic Variation in Animals. Intech, Rijeka, Croatia.

Evren K. 2004. Genetic Diversity of Native and Crossbreed Sheep Breeds in Anatolia. A thesis submitted to the graduate school of natural and applied sciences of Middle East Technical University. December 2004, 125 pages.

FAO. 2000. Conserving and developing farm animal diversity. In: The State of the World: Animal Genetic Resources for Food and Agriculture. Food and Agricultural Organization, Rome.

FAO. 2011. Guidelines on Molecular Genetic Characterization of Animal Genetic Resources. Food and Agricultural Organization, Rome.

Farid A, O'Reilly E, Dollard C, Kelsey Jr. CR. 2000. Genetic analysis of ten sheep breeds using microsatellite markers. Canadian J Anim Sci 80 (1): 9-17. 
Frankham R, Briscoe DA, Ballou JD. 2002. Introduction to Conservation Genetics. Cambridge University Press, New York, USA.

Gizaw S, Komen H, Hanotte O, van Arendonk J.A.M. 2008a. Indigenous sheep resources of Ethiopia: types, production systems and farmers preferences. Anim Genet Resour Inform 43: 25-40.

Gizaw S. 2008. Sheep Resources of Ethiopia: Genetic diversity and breeding strategy. [Dissertation]. Wageningen University, The Netherlands.

Groeneveld LF, Lenstra JA, Eding H, Toro MA, Scherf B, Pilling D, Negrini R, Finlay EK, Jianlin H, Groeneveld E, Weigend S, the GLOBALDIV Consortium. 2010. Genetic diversity in farm animals-a review. Anim Genet 41: 6-31.

Hailu D, Tibbo M, Takahashi Y, Nomura K, Hanada H, Amano A. 2008. Microsatellite analysis reveals high genetic diversity but low genetic structure in Ethiopian indigenous cattle populations. Anim Genet 39: 425-431.

Halima H, Lababidi S, Rischkowsky B, Baum M, Tibbo M. 2012b. Molecular characterization of Ethiopian indigenous goat populations. Trop Anim Health Prod 44 (6): 1239-1246.

Hannotte O, Jialin H. 2005. Genetic characterization of livestock populations and its use in conservation decision making. In: Ruane $\mathbf{J}$, Sannino A (eds.). The Role of Biotechnology in Exploring and Protecting Genetic Resources, Rome, Italy.

Hatami GF, Khomeini M, Mehran P. 2014. Genetic variation among pelt sheep population using microsatellite markers. Intl J Anim Breed Genet 2 (1): 55-62.

Hirbo J, Muigai A, Naqvi AN, Rege ED, Hanotte O. 2006. The Genetic Diversity of Trans-caucasian Native Sheep Breeds. Asian-Aust J Anim Sci 19 (7): 943-952.

Hoda A, Marsan PA. 2012. Genetic characterization of Albanian sheep breeds by microsatellite markers. In: Caliskan M (ed.). Biochemistry, Genetics and Molecular Biology: Analysis of Genetic Variation in Animals. Intech, Rijeka, Croatia.

Kalinowski ST. 2004. Counting alleles with rarefaction: Private alleles and hierarchical sampling designs. Conserv Genet 5: 539-543.

Kavitha A, Vijayalakshmi M, Sudhakar P, Narasimha G. 2010. Screening of Actinomycete strains for the production of antifungal metabolites. African J Microbiol Res 4 (1): 27-32.

Mahmoud AH, Swelum A, Farah MA, Alanazi K, Rady A, Salah M, Amor N, Mohammed O. 2017. Genetic diversity among Sawakni, Berberi and Najdi sheep breeds in Saudi Arabia using microsatellites markers. African J of Biotech.

Mahmoudi B, Babayev MSh, Hayeri Khiavi F, Pourhosein A, Daliri M. 2011. Breed characteristics in Iranian native goat populations. J Cell Anim Biol 5 (7): 129-134.
Mahmud (2010). Present status of the world goat populations and their productivity. Lohmann Information 45 (2): 42-53.

Marshall TC, Slate J, Kruuuk LEB, Pemberton JM. 1998. Statistical confidence for likelihood-based paternity inference in natural population. Mol Ecol 7: 639-655.

Mekuriaw G, Gizaw S, Dessie T, Mwai O, Djikeng A, Tesfaye K. 2016. A Review on Current Knowledge of Genetic Diversity of Domestic Goats (Capra hircus) Identified by Microsatellite Loci: How those Efforts are Strong to Support the Breeding Programs? J Life Sci Biomed 6 (2): 22-32.

Mukhongo D. M, Mwai O, M Tapio M, Muigai A. 2014. Genetic Diversity and Population Structure of the Indigenous Sheep in Kenya Based on Microsatellite Analysis: Implications for their Conservation. Journal of Livestock Science.

Nanekarani S, Amirinia C, Amirmozafari N. 2011. Genetic analysis of Karakul sheep breed using microsatellite markers. African J Microbiol Res 5 (6): 703-707.

Nigussie H. 2015. Phenotypic and Genetic Characterization of Indigenous Sheep Breeds of Eastern Ethiopia. A Dissertation Submitted to the School of Animal and Range Sciences, School of Graduate Studies, March 2015, Haramaya University, Haramaya, Ethiopia.

Ojango JM, Mpofu N, Marshall K, Andersson-Eklund L. 2011. Quantitative methods to improve the understanding and utilisation of animal genetic resources In: Animal Genetics Training Resource, version 3. Ojango JM, Malmfors B, Okeyo AM. (eds). International Livestock Research Institute, Nairobi, Kenya, and Swedish University of Agricultural Sciences, Uppsala, Sweden.

Pramod S, Rasberry AB, Butler TG, Welch ME. 2011. Characterization of long transcribed microsatellites in Helianthus annuus (Asteraceae). Am J Bot 98 (12): e388-390. DOI: 10.3732/ajb.1100232.

Sithembile. 2011. Genetic and phenotypic characterization of the South African Namaqua Afrikaner sheep breed. [Thesis]. Department of Animal and Wildlife Sciences, University of Pretoria, Pretoria, South Africa.

Toro MA, Fernandez J, Caballero A. 2009. Molecular characterisation of breeds and its use in conservation. Livestock Sci 120: 174-194.

Wollny CBA. 2003. The need to conserve farm animal genetic resources in Africa: should policy makers be concerned? Ecol Econ 45: 341351.

Yilmaz O, Sezenler T, Sevim S, Cemal I, Karaca O, Yaman Y, Karadag O. 2015. Genetic relationships among four Turkish sheep breeds using microsatellites. Turk J Vet Anim Sci 39: 576-582

Zeng XC, Chen HY, Hui WQ, Jia B, Du YC, Tian YZ. 2010. Genetic diversity measures of 8 local sheep breeds in Northwest of China for genetic resource conservation. Asian-Aust J Anim Sci 23 (12): 15521556. 